\title{
MATCHFUNDING: FINANCIANDO LA TRANSICIÓN AGROECOLÓGICA (PÓSTER)
}

\author{
González-Azcárate, Mario**, Cruz-Maceín , José Luis
}

Instituto Madrileño de Investigación y Desarrollo Rural, Agrario y Alimentario (Madrid, mario.gonzalez@madrid.org)

\section{Resumen}

El Matchfunding es un mecanismo de micromecenazgo (crowdfunding) a través del cual los ciudadanos en colaboración con los gobiernos subnacionales pueden financiar proyectos que tengan un impacto local positivo.

En este sentido, y dentro de la estrategia de promover y apoyar el desarrollo rural y la agricultura sostenible en la región de Madrid, el Grupo Operativo Madrid KmRegión ha lanzado tres convocatorias del proyecto "Matchfunding Madrid Km Región". Estas convocatorias están dirigidas a proyectos, nuevos o existentes, del canal corto de comercialización de alimentos de la región de Madrid que quieran realizar inversiones innovadoras y adquirir nuevos vínculos de fidelización con los consumidores.

Los resultados sugieren que el matchfunding puede ser una herramienta útil para los gobiernos locales y regionales que quieran impulsar la creación y la expansión de iniciativas agroecológicas que contribuyan al establecimiento de un sistema alimentario local más sostenible en sus regiones. Este mecanismo puede ser eficaz para redefinir los sistemas de subvención públicos actuales y atajar los problemas financieros del sector agroecológico de pequeña escala.

La presente comunicación tiene como objetivo exponer la naturaleza, dinámica y resultados del "Matchfunding Madrid Km Región", así como los potenciales beneficios de esta herramienta para los gobiernos subnacionales, productores y consumidores.

\section{Palabras claves}

"crowdfunding", "matchfunding", "civic crowdfunding"," canal corto de comercialización", "sistema alimentario local"

\section{Introducción}

El crowdfunding cívico es un mecanismo de microfinanciación (crowdfunding) a través del cual los ciudadanos en colaboración con las entidades públicas y privadas pueden financiar proyectos que beneficien a la comunidad local [Charbit et al., (2017)]. Dentro de la categoría crowdfunding cívico, el matchfunding es una modalidad donde las instituciones públicas o privadas aportan fondos adicionales a las campañas de crowdfunding, fomentando las donaciones privadas y aumentando la cantidad de dinero recaudado [Baeck et al. (2017); European Crowdfunding Network, (2018)].

En este sentido, y dentro de la estrategia de promover y apoyar el desarrollo rural y la agricultura sostenible en la región de Madrid, el Grupo Operativo Madrid KmRegión ha lanzado tres convocatorias del proyecto "Matchfunding Madrid Km Región". Estas convocatorias estaban dirigidas a proyectos, nuevos o existentes, del canal corto de alimentos de la región de Madrid que quisieran realizar inversiones innovadoras y adquirir nuevos vínculos de fidelización con los consumidores. Los fondos institucionales doblaron una parte de lo recaudado mediante donaciones privadas en campañas de crowdfunding. Para liberar estos fondos, las campañas de crowdfunding debían alcanzar un objetivo mínimo de recaudación y un número mínimo de donantes, asegurando así la viabilidad y el interés público de los proyectos cofinanciados. El objetivo de esta investigación es analizar el potencial del matchfunding como herramienta para apoyar el desarrollo rural y la transición agroecológica desde los gobiernos autonómicos y locales mediante el análisis del estudio de caso "Matchfunding Madrid Km Región”.

\section{Metodología}

Los métodos empleados en la investigación son mixtos. Primero, la observación participante durante todo el preparación y lanzamiento de las convocatorias de matchfunding. Además, se realizó un análisis descriptivo de los datos de la plataforma de crowdfunding después de cada campaña. Este análisis fue complementado con entrevistas a los promotores de los proyectos, una encuesta a los donantes de los proyectos y entrevistas con los responsables políticos de 35 municipios de la Comunidad de Madrid. 


\section{Resultados}

En total 43 proyectos se presentaron a las convocatorias de matchfunding, de los cuales 13 fueron seleccionados. La tipología de proyectos seleccionados fue muy variada: un supermercado cooperativo, un centro logístico para las productoras locales, un sistema de reparto de alimentos locales en bici, etc. Los promotores de los proyectos seleccionados recibieron capacitación sobre cómo llevar a cabo una campaña de crowdfunding de manera efectiva. Todas las campañas de crowdfunding fueron exitosas. En total se han recaudado en torno a 80.000 euros que han sido complementados con 38.000 euros de fondos institucionales.

Los ciudadanos tenían la opción de donar una cantidad libre de dinero o de aportar una cantidad asociada a una recompensa, la cual podía ser una preventa de productos alimentarios, una visita guiada a los proyectos o un agradecimiento público en las redes sociales entre otras. La cantidad media donada fue de $90 €$ y la moda $40 €$. Las campañas de crowdfunding duraban 40 días y fueron más activas al principio y al final, trascurriendo un periodo valle más inactivo entre ambos periodos.

Según los promotores, el matchfunding ha supuesto un cambio de nivel y el afianzamiento de los proyectos financiados. Además, han expandido sus redes de clientes, generado vínculos más profundos con ellos y aprendido sobre nuevas herramientas de marketing. Las campañas suponen un esfuerzo considerable, pero les ha compensado a la vista de los resultados obtenidos. Por ello, todos volverían a hacer una campaña de matchfunding o crowdfunding si tuviesen que acceder a más financiación en un futuro.

Un tercio de los donantes tenía una relación comercial previa con el proyecto, otro tercio una relación personal previa con el promotor/es y el último tercio no conocía previamente el proyecto. Las motivaciones más importantes fueron la temática del proyecto (los canales cortos de comercialización), la información recibida sobre el proyecto y el hecho de apoyar un proyecto que beneficiase a la comunidad local. La distancia mediana entre el domicilio del donante y el proyecto fue de 10 kilómetros, lo que resalta el localismo de este mecanismo de financiación.

Los miembros de los ayuntamientos mostraron un gran interés por este mecanismo, considerándolo una herramienta con gran potencial para financiar iniciativas relacionadas con los canales cortos de comercialización. Las principales barreras percibidas están relacionadas con la falta de conocimiento sobre el mecanismo de matchfunding y sobre su encaje administrativo y legislativo.

\section{Conclusiones}

El matchfunding genera una serie de beneficios en distintos aspectos como la facilitación de las alianzas público-privadas, el fomento de la participación ciudadana, la mejora de la eficiencia del gasto de recursos públicos o la generación de conciencia ciudadana entre otros [Cruz.Maceín y González-Azcárate, (2020)]. Por ello, el matchfunding puede ser una herramienta de gran utilidad para los gobiernos locales y regionales que quieran apoyar el desarrollo rural mediante la creación y la expansión de iniciativas que contribuyan al establecimiento de un sistema alimentario más sostenible en sus regiones. Además, este mecanismo puede ser eficaz para redefinir los sistemas de subvención públicos actuales y atajar los problemas financieros del sector agroecológico de pequeña escala. Por otro lado, los principales retos a los que se enfrenta el escalamiento de estas iniciativas son la falta de conocimiento sobre el mecanismo, así como la limitada capacidad de adaptación de las administraciones públicas en temas de financiación.

\section{Bibliografía}

Baeck, P., Bone, J., y Mitchell, S. (2017). Matching the crowd: Combining crowdfunding and institutional funding to get great ideas off the ground. Nesta, London.

Charbit, Claire y Desmoulins, G. (2017). Civic Crowdfunding: A Collective Option For Local Public Goods? OECD Regional Development Working Papers.

Cruz-Maceín, J.L. y González-Azcárate, M. (2020). Innovación social y políticas públicas locales. Matchfunding, ¿una nueva herramienta para el desarrollo local?, Series estudios IMIDRA 5. Instituto Madrileño de Investigación y Desarrollo Rural Agrario y Alimentario, Comunidad de Madrid.

European Crowdfunding Network: Francesca Passeri (ed.) (2018), Triggering Participation: A Collection of Civic Crowdfunding and Match-funding Experiences in the EU, Brussels.

\section{Agradecimientos}

Mario González-Azcárate está contratado en el marco del programa de ayudas para la formación de personal investigador del IMIDRA. 